\title{
$\langle$ 无损检测〉
}

\section{基于单尺度 Retinex 与改进的 $K$-均值聚类的 涡流热成像缺陷检测}

\author{
张庆宇 ${ }^{1,2}$, 范玉刚 ${ }^{1,2}$, 高 阳 ${ }^{1,2}$ \\ (1. 昆明理工大学 信息工程与自动化学院, 云南 昆明 650500 ; \\ 2. 云南省矿物管道输送工程技术研究中心, 云南 昆明 650500)
}

\begin{abstract}
摘要: 在利用涡流红外热成像技术检测金属材料损伤缺陷时, 因热波属于衰减波, 且热波三维热扩 散等问题, 导致采集的红外图像中缺陷部位模糊。针对该问题, 提出一种基于单尺度 Retinex 与改 进的 $K$-均值聚类的缺陷检测方法, 用于处理红外图像特征增强、图像分割和边缘特征提取等问题。 该方法首先利用单尺度 Retinex（single-scale Retinex, SSR）对红外热图像进行图像增强, 强化缺陷 特征, 然后利用改进的 $K$-均值聚类算法对图像进行分割, 最后采用数学形态学算法处理图像, 去除 缺陷图像中无用信息, 并利用 Canny 算子检测出缺陷边缘。实验结果证明, 该方法有效地检测出金 属材料试件缺陷, 并提取出完整清晰的缺陷边缘。
\end{abstract}

关键词: 涡流红外热成像; 单尺度 Retinex; 图像增强; $K$-均值聚类; 数学形态学; Canny 算子 中图分类号: TN215 文献标志码：A 文章编号：1001-8891(2020)10-1001-06

\section{Defect Detection of Eddy-Current Thermography Based on Single-Scale Retinex and Improved $K$-means Clustering}

\author{
ZHANG Qingyu $^{1,2}$, FAN Yugang ${ }^{1,2}$, GAO Yang ${ }^{1,2}$
}

(1. College of Information Engineering and Automation, Kunming University of Science and Technology, Kunming 650500, China;

2. Yunnan Institute of Mineral Pipeline Engineering Technology, Kunming 650500, China)

\begin{abstract}
When eddy-current infrared thermal-imaging technology is used to detect metal-material damage defects, the infrared image is susceptible to noise and may also contain useless information, which can result in blurring of damage defects. To address this problem, a defect-detection method based on single-scale Retinex and improved $K$-means clustering is proposed to perform infrared image-feature enhancement, image segmentation, and edge feature extraction. First, the image is enhanced using single-scale Retinex. Additionally, the defect features are enhanced. Then, an improved K-means clustering algorithm is used to segment the image. Finally, a mathematical morphology algorithm is used to process the image, which removes the useless information in the defective image and uses a Canny operator to detect the defect edge. The experimental results show that the method effectively detects defects of metal-material specimens and extracts complete and clear defect edges of the metal-material specimens.
\end{abstract}

Key words: eddy current infrared thermal imaging, single-scale Retinex, image enhancement, $K$-means clustering, mathematical morphology, Canny operator

\section{0 引言}

收稿日期: 2019-07-15; 修订日期: 2019-08-23.

作者简介: 张庆宇 (1995-), 男, 硕士研究生, 河北省期鄲市人, 主要从事故障诊断、图像处理。E-mail: 280208691@qq.com。

通信作者: 范玉刚（1963-）, 男, 副教授, 山东省威海市人, 主要从事故障诊断、数据挖掘。E-mail: ygfan@qq.com。

基金项目：国家自然科学基金（61741310）。
金属材料部件内部会产生裂纹和分层等损伤缺 陷, 这些缺陷影响设备的正常运行, 形成生产安全 
隐患, 并威胁工作人员的人身安全。因此, 对设备 关键金属部件进行无损检测, 并判定设备运行状态 有着重要意义。作为一种无损检测技术，脉冲浴流 热成像检测技术利用电磁感应原理对金属材料试件 加热, 并对试件红外热图像进行分析处理, 其具有 非接触性 ${ }^{[1]}$ 、实时性和准确性等优点, 被广泛应用 于金属材料的损伤检测。例如文献 [2]通过浴流加 热得到钢板的红外热图像, 利用超像素结合模糊 $\mathrm{C}$ 均值分割得到缺陷图像, 但其限制试件与红外热成 像仪的距离。为了增强图像的清晰度, 马忠丽 ${ }^{[3]}$ 等学者利用 SSR (single-scale Retinex, SSR) 算法 融合边缘信息, 解决海雾对图像带来的模糊影响; 李卫东 ${ }^{[4]}$ 等学者在 SSR 算法中, 利用快速引导滤波 代替高斯滤波, 在保持图像整体的同时也保留了细 节部分。针对图像的分割问题, Md Nasim Reza ${ }^{[5]}$ 通过 $K$-均值聚类对水稻米粒区域的 Lab 图像进行分 割; 文献[6]采用超像素聚类来引导 $K$-均值聚类, 有 效地分割叶子图像中病害部分。为了提取连续的边 缘, Kumar ${ }^{[7]}$ 等人采用 Canny 算子准确地识别出预 定义的像素组, 有效地检测目标图像的边缘; 文献 [8]利用灰度变换结合 Canny 的边缘检测方法, 改善 了不连续成像问题; 但 Canny 敏感性较高, 检测出 缺陷边缘的同时也包含了无用信息; 杨信廷 ${ }^{[9]}$ 等学 者采用数学形态学进行图像校正, 避免无用信息对 边缘检测的影响, 提高边缘的质量。上述研究涉及 的图像特征增强、图像分割和边缘特征提取等图像 处理技术，是基于红外热成像金属材料损伤检测问 题的重要研究内容。

由于图像种类过多, 不同图像有不同的分割与 边缘检测方法, 没有通用的方法对所有图像进行处 理 ${ }^{[10]}$ 。金属材料试件受锻造工艺影响, 存在厚度不 均匀和表面不平滑情况, 导致局部温度异常, 红外 图像局部与缺陷颜色相似, 造成了缺陷定位、分割、 特征提取的困难。针对上述问题, 本文利用 SSR 算 法突出红外热图像中缺陷部分, 通过改进后的 $K-$ 均值聚类分割图像, 并采用数学形态学结合 Canny 算子方法, 去除缺陷部分图像中的无用信息, 并提 取边缘特征，检测出金属材料试件缺陷。

\section{1 涡流热成像缺陷检测}

\section{1 涡流热成像图像增强}

\subsubsection{Retinex 理论}

Retinex 理论由 Edwin. H. Land ${ }^{[11]}$ 在 1963 年提 出, 是一种以色感一致性为基础的图像增强方法。 其基本内容是物体的颜色由物体对长波、中波和短
波光线的反射能力决定, 即反射分量 $R$ 由输入图像 $S(x, y)$ 决定, 降低或去除入射分量 $L$ 对输入图像 $S(x, y)$ 的影响。具体原理如下:

$$
L(x, y) \cdot R(x, y)=S(x, y)
$$

式中: $(x, y)$ 为输入图像的每个点; $L(x, y)$ 为入射图像; $R(x, y)$ 为反射图像; $S(x, y)$ 为输入图像。

\subsubsection{SSR 算法红外热图像增强}

通过 SSR 算法增强红外热图像较为明亮部分, 减弱较暗部分, 为准确地检测出金属材料试件的缺 陷提供支撑，降低误分割的机率。

SSR 算法是 Retinex 理论中的基础算法, 算法 流程如下:

(1)对反射图像 $R(x, y)$ 、入射图像 $L(x, y)$ 和浴流热 图像 $S(x, y)$ 取对数, 将反射光分量 $R$ 和入射光分量 $L$ 分离, 即:

$$
\lg R(x, y)+\lg L(x, y)=\lg S(x, y)
$$

(2)利用高斯卷积函数 $G(x, y)$ 对浴流热图像 $S(x, y)$ 进行卷积, 得到卷积后的图像 $D(x, y)$, 即:

$$
S(x, y) \cdot G(x, y)=D(x, y)
$$

(3)对流热图像 $S(x, y)$ 减去卷积后的图像 $D(x, y)$ 取倒数, 并相减得到高频增强的图像 $F(x, y)$, 即:

$$
\lg S(x, y)-\lg D(x, y)=F(x, y)
$$

(4)取高频增强的图像 $F(x, y)$ 的反对数, 得到增 强后的图像 $P(x, y)$, 即:

$$
\exp F(x, y)=P(x, y)
$$

\section{2 基于改进的 $K$-均值聚类的图像分割}

本文在 SSR 增强红外热图像的基础上, 采用 $K$-均值聚类算法对红外热图像进行分割。 $K$-均值聚 类算法由 Macqueen ${ }^{[12]}$ 首次提出, 它是一种通过迭 代寻找局部最优划分的无监督算法。因聚类中心的 数量对分割的结果起到决定性作用, 为此加入标准 差衡量不同聚类中心数量时每一聚类的所有像素值 离散程度与关联度, 标准差越小，则该聚类中的所 有像素值越相近。其具体过程如下:

(1) 随机选取 $K$ 个像素点 $O_{K}, K=1,2, \cdots, N$ 作为 初始的聚类中心，每个聚类中心代表一个聚类;

(2)计算所有聚类中每个像素点 $X_{i}$ 与聚类中心的 欧式距离 $D:$

$$
D=\sqrt{\sum_{i=1}^{n}\left(O_{K}-X_{i}\right)^{2}}
$$

式中: $n$ 为第 $K$ 个聚类中像素点的数量。

(3)将所有像素点划分给离其距离最近的聚类中 心, 当所有像素点被分配完毕, 则会在每个聚类中 重新计算 $N$ 次该聚类的聚类中心 $P_{K}, K=1,2, \cdots, N$, 直到每个聚类的误差平方和 $S$ 达到局部最小。 
(4)计算 $K$ 个聚类中心时每个聚类的标准差, $K$ $=1,2, \cdots, N$ 。

(5)若第 $N+1$ 个聚类中心所有聚类的标准差的最 小值大于或等于第 $N$ 个聚类中心所有聚类的标准差 的最小值则停止运算, 选取 $N$ 为最优聚类中心数量。

利用标准差确定每一聚类中的像素关联度, 从 而选取最优聚类中心数量, 以避免图像的误分割, 提高分割精度。但分割后的图像存在无用干扰信息, 影响缺陷的边缘特征的提取, 因此分割后的图像仍 需要去干扰处理才能得到更好的边缘检测结果。

\section{3 缺陷边缘检测}

由于分割后的红外热图像存在无用信息, 而 Canny 算子 ${ }^{[13]}$ 在检测出缺陷边缘的同时也包含了这 些信息。因此, 采用数学形态学对被分割出的缺陷 部分图像进行处理, 保证边缘检测的准确性。

腐蚀与膨胀是数学形态学中的最基本运算 ${ }^{[14]}$, 该运算使图像的边界收缩, 以达到消除部分干扰的 目的。腐蚀的具体过程为, 用某个结构元素 $B$ 对分 割后的红外热图像 $A$ 进行探测, 以便找出在图像内 部可放下结构元素 $B$ 的区域, 表示为 $A \Theta B$ 。该方法 定义为:

$$
A \Theta B=\left\{x, y \mid(B)_{x y} \subseteq A\right\}
$$

膨胀是腐蚀运算的对偶运算, 该运算使图像的 边界扩充, 以达到填补目标区域的空洞目的。膨胀 的具体过程为, 用结构元素 $B$ 的所有点平移分割后 的红外热图像 $A$, 然后计算其并集得到最终结果, 表示为 $A \oplus B$ 。该方法定义为:

$$
A \oplus B=\left\{x, y \mid(B)_{x y} \cap A \neq \Phi\right\}
$$

式中: $\oplus$ 为空集。

利用开运算平滑图像边缘, 去除无用信息, 为 Canny 算子的边缘检测提供支撑。开运算的过程为 先对分割后的红外热图像 $A$ 进行腐蚀, 然后对腐蚀 后的图像进行膨胀, 表示为 $A \bigcirc B$ 。该方法定义为:

$$
A \bigcirc B=(A \Theta B) \oplus B
$$

缺陷图像经数学形态学处理后, 消除了无用信 息, 利用 Canny 算子提取图像的边缘特征, 实现缺 陷的检测。

通过以上分析, 可得浴流热成像缺陷检测流程, 如图 1 所示, 首先通过 SSR 算法增强红外热图像, 突出图像中的缺陷部分; 然后利用 $K$-均值聚类分割 图像, 得到缺陷部分的图像; 最后利用数学形态学 结合 Canny 算子, 去除缺陷部分图像无用信息并提 取边缘特征。

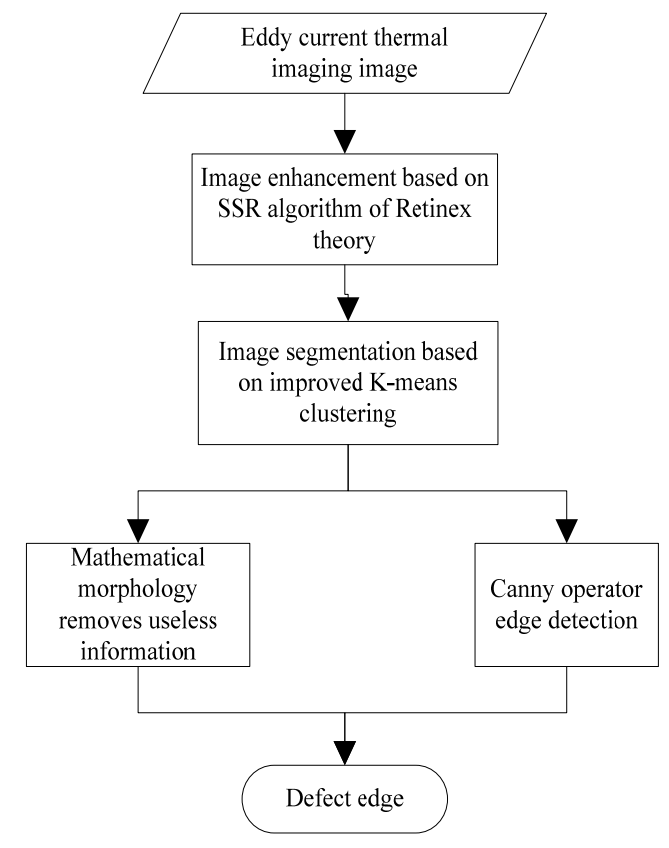

图 1 涡流热成像缺陷检测方法流程

Fig.1 Eddy current thermography defect detection method flow

\section{2 实验结果与分析}

涡流热成像实验平台由浴流加热装置（如图 2 所示) 和红外热像仪组成, 被测金属材料试件如图 3 所示。被测金属试件为铁板, 试件尺寸为 $100 \mathrm{~mm} \times 80$ $\mathrm{mm} \times 5 \mathrm{~mm}$, 缺陷尺寸为 $10 \mathrm{~mm} \times 1.5 \mathrm{~mm} \times 5 \mathrm{~mm}$; 红外热像仪采用金思特 HT- 18 , 其分辨率为 220 pixel $\times 160$ pixel, 测温灵敏度为 $0.07^{\circ} \mathrm{C}$ 。在调试好 浴流加热装置之后, 对被测金属材料试件进行浴流 加热, 加热 $8 \mathrm{~s}$ 后进行图像实时采集。

图 4(a)为红外热成像采集的试件红外热图像, 对缺陷面的试件进行浴流激励时, 因缺陷部位被空 气填充, 浴流在此断开, 无法对缺陷部分加热, 导 致缺陷部分温度低, 图像的缺陷部分出现异常。对 比图 4(a)与图 4(b), 被测金属材料试件的热成像图 像经 SSR 算法增强后, 其较亮部分被增强, 较弱部 分被减弱, 增强了图像的对比度, 使缺陷部分更加 清晰。

将 SSR 算法增强后的图像转换为灰度图, 然后 通过比较不同聚类中心数量时的每个聚类标准差中 的最小值确定聚类中心数量, 如表 1 所示, 当聚类 中心数量为 5 时, 所有聚类标准差中最小值为 0.0318 , 等于聚类中心数量为 6 时的所有聚类标准 差中最小值, 故最优聚类中心数量为 5 , 分割结果 如图 5 所示。对比图 6(a)与(b), 经 SSR 增强后, 图 像中的缺陷部分得到增强。 


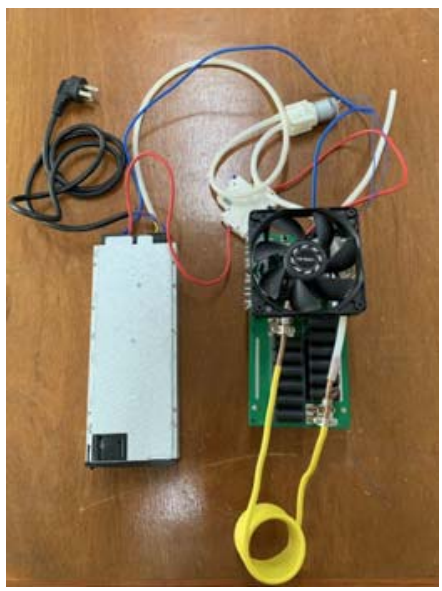

图 2 浴流加热装置

Fig.2 Eddy current heating device

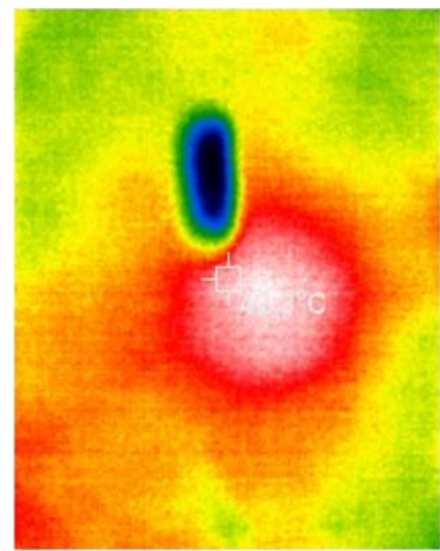

(a) 试件红外热图像 (a) Test piece infrared thermal image

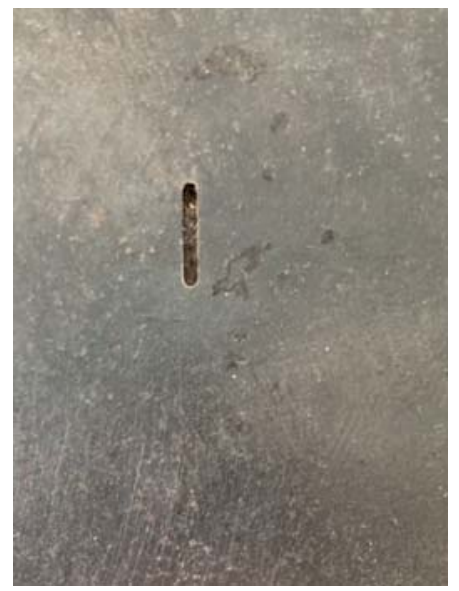

图 3 被测金属材料试件

Fig.3 Tested metal material test piece

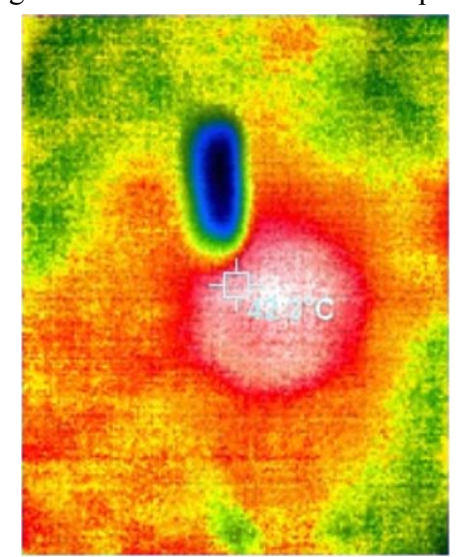

(b) SSR 增强后的图像 (b) Enhanced image of SSR

图 4 SSR 增强前后对比图像

Fig.4 Contrast image before and after SSR enhancement

表 1 聚类标准差

Table 1 Cluster standard deviation

\begin{tabular}{cc}
\hline $\begin{array}{c}\text { Number of } \\
\text { cluster centers }\end{array}$ & $\begin{array}{c}\text { The smallest value } \\
\text { among all cluster } \\
\text { standard deviations }\end{array}$ \\
\hline 2 & 0.3293 \\
3 & 0.2477 \\
4 & 0.1923 \\
5 & 0.0318 \\
6 & 0.0318 \\
\hline
\end{tabular}

由于分割后的图像存在无用信息, 因此采用数 学形态学进行处理, 如图 7(a)所示; 最后对处理后 的缺陷图像进行 Canny 算子的边缘检测, 如图 8(a) 所示。对比图 7(a)和(b), 图 8(a)和(b)可以发现, 没 有经过 SSR 增强后的图像, 缺陷部分的边缘出现不 完整的现象; Canny 算子虽然识别出了所有的缺陷
边缘, 但也同时包含大量无用信息, 如图 8(c)所示, 会在一定程度上干扰对缺陷的识别。

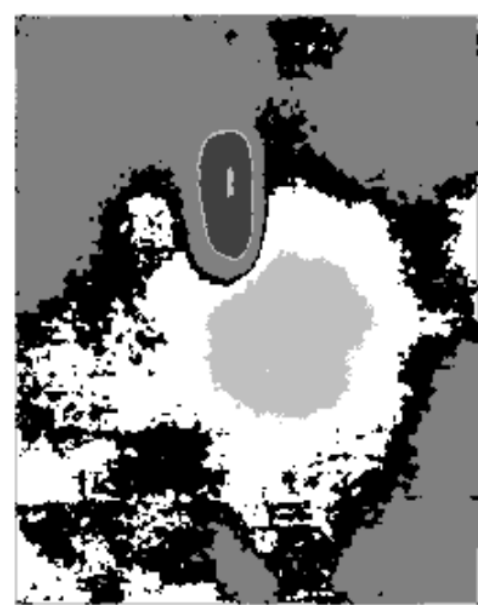

图 5 分割增强后的图像

Fig.5 Split enhanced image 


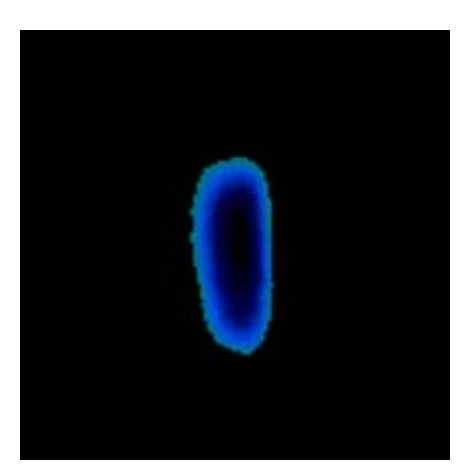

(a) 增强后的缺陷部分图像 (a) Enhanced defect part image

图 6 增强前后缺陷部分对比图像

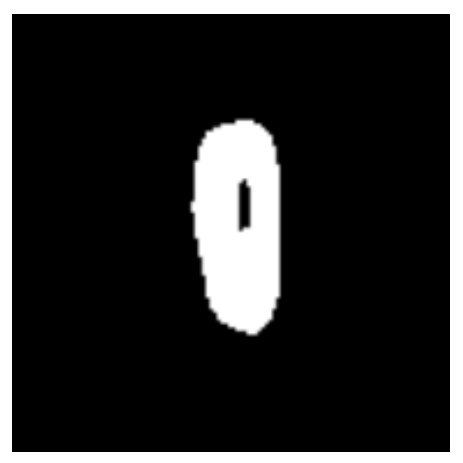

(a) 数学形态学处理增强后的缺陷部分图像

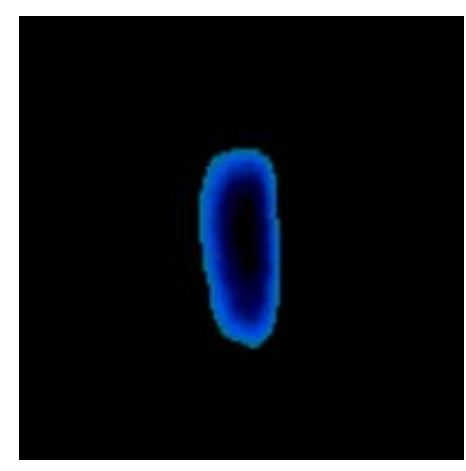

(b) 原图像的缺陷部分图像 (b) Defective image of the original image

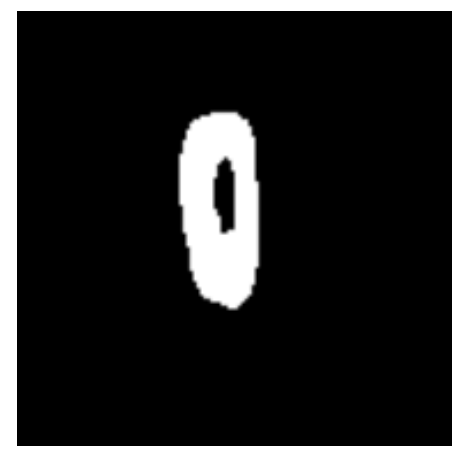

(b) 数学形态学处理原图像的缺陷部分图像

(a) Mathematical morphology processing enhanced defect image (b) Mathematical morphology processing defective image of original image

图 7 数学形态学处理增强前后的缺陷对比图像

Fig.7 Mathematical morphology processing defect contrast images before and after enhancement

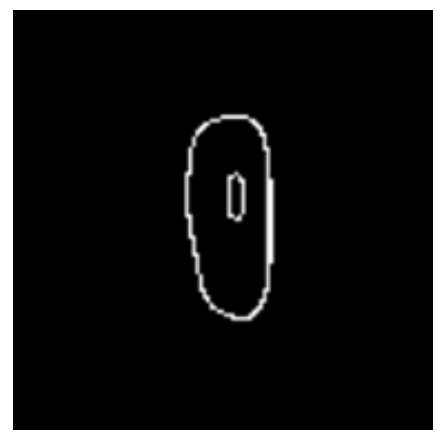

(a) 本文方法得到的缺陷边缘

(a) The edge of the defect obtained by the method of this paper

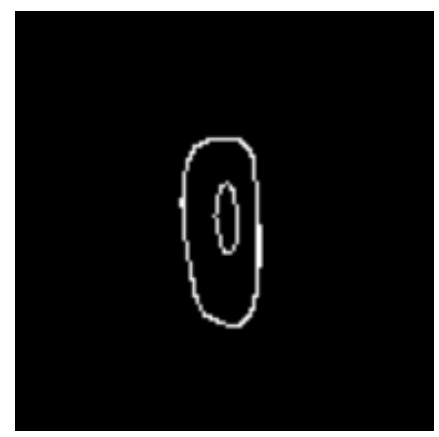

(b) 原图像缺陷边缘

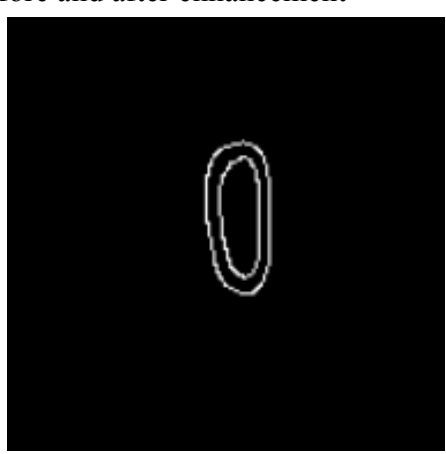

(c) 没有经过数学形态学处理的缺陷边缘

(b) The edge of the defective portion (c) The edge of the defective portion that has of the original image not been mathematically processed

Fig.8 Experimental result comparison image

\section{3 结论}

本文利用 SSR 算法对属材料试件红外热图像 进行增强, 然后通过标准差衡量聚类中所有像素的 关联度, 从而选取最优 $K$-均值聚类的聚类中心数 量, 以避免缺陷图像的误分割, 提高分割精度; 最 后通过数学形态学去除缺陷部分图像中的无用信
息, 实现了对金属试件缺陷的检测, 且提取到清晰 的缺陷边缘。本文方法解决了红外热图像分割后, 缺陷部位模糊、且存在无用信息等问题, 实现了缺 陷边缘精准提取。

\section{参考文献:}

[1] 顾桂梅, 刘丽, 贾文晶. 一种钢轨裂纹红外图像处理算法研究 $[\mathrm{J}]$. 铁道学报, 2018, 40(11): 129-133. 
GU Guimei, LIU Li, JIA Wenjing. Research of processing algorithms for rail crack infrared image[J]. Journal of the China Railway Society, 2018, 40(11): 129-133.

[2] XU Changhang, Xie Jing, Chen Guoming, et al. An infrared thermal image processing framework based on superpixel algorithm to detect cracks on metal surface[J]. Infrared Physics \& Technology, 2014, 67: 266-272.

[3] 马忠丽, 文杰. 融合边缘信息的单尺度 Retinex 海雾去除算法 [J]. 计算机辅助设计与图形学学报, 2015, 27(2): 217-225.

MA Zhongli, WEN Jie. Single-scale Retinex sea fog removal algorithm fused the edge information[J]. Journal of Computer-Aided Design \& Computer Graphics, 2015, 27(2): 217-225.

[4] 李卫东, 彭琪. 基于混合映射的高动态范围场景再现 $[\mathrm{J}]$. 光学精 密工程, 2018, 26(12): 3051-3059.

LI Weidong, PENG Qi, High-dynamic range scenes reproduction based on hybrid mapping[J]. Optics and Precision Engineering, 2018, 26(12): 3051-3059.

[5] Md Nasim Reza, In Seop Na, Sun Wook Baek, et al. Rice yield estimation based on K-means clustering with graph-cut segmentation using low-altitude UAV images[J]. Biosystems Engineering, 2019, 177: $109-121$.

[6] ZHANG Shanwen, WANG Haoxiang, HUANG Wenzhun. Plant diseased leaf segmentation and recognition by fusion of superpixel[J]. K-means and PHOG. Optik, 2018, 157: 866-872.

[7] Kumar Gaurav, Umesh Ghanekar. Image steganography based on Canny edge detection, dilation operator and hybrid coding $[\mathrm{J}]$. Journal of Information Security and Applications, 2018, 41: 41-51.
[8] DI Haibin, GAO Dengliang. Gray-level transformation and Canny edge detection for 3D seismic discontinuity enhancement[J]. Computers \& Geosciences, 2014, 72: 192-200.

[9] 杨信廷, 孙文娟, 李明. 基于 $\mathrm{K}$ 均值聚类和开闭交替滤波的黄瓜叶 片水滴苂光图像分割[J]. 农业工程学报, 2016, 32(17): 136-143.

YANG Xinting, SUN Wenjuan, LI Ming. Water droplets fluorescence image segmentation of cucumber leaves based on K-means clustering with opening and closing alternately filtering[J]. Transactions of the Chinese Society of Agricultural Engineering, 2016, 32(17): 136-143.

[10] 余成波, 曾亮, 张林. 基于 OTSU 和区域生长的电气设备多点故 障分割[J]. 红外技术, 2018, 40(10): 1008-1012.

YU Chengbo, ZENG Liang, ZHANG Lin. Multi point fault segmentation of electrical equipment based on OTSU and region growth[J]. Infrared Technology, 2018, 40(10): 1008-1012.

[11] Land E, Mc Cann J. Lightness and Retinex theory[J]. Journal of the Optical Society of America, 1971, 61(1): 1-11.

[12] Macqueen J. Some methods for classification and analysis of multi variate observations $[\mathrm{C}] / /$ Proc. of Berkeley Symposium on Mathematical Statistics \& Probability, 1965(1): 281-297.

[13] Canny J. A computational approach to edge detection[J]. IEEE Transactions on Pattern Analysis and Machine Intelligence, 1986, 8(6): 679-698.

[14] 王宁, 陈方, 于博. 基于形态学开运算的面向对象滑坡提取方法 研究 [J]. 遥感技术与应用, 2018, 33(3): 520-529.

WANG Ning, CHENG Fang, YU Bo. A object-oriented landslide extraction method based on morphological opening operation[J]. Remote Sensing Technology and Application, 2018, 33(3): 520- 529. 